\title{
Multiple-layer Closure of Myoma Bed in Laparoscopic Myomectomy
}

\author{
${ }^{1}$ Nutan Jain, ${ }^{2}$ Sonam Singh
}

\section{ABSTRACT}

Objective: To assess the feasibility and outcome of laparoscopic myomectomy and multiple-layer closure of myoma bed for management of myomas at a tertiary care hospital.

Materials and methods: Five hundred and ten patients from January 2011 to January 2017 with large and moderate size myomas were managed by laparoscopic myomectomy. Indications were subfertility, menorrhagia, and abdominal mass. Preoperative evaluation included history, clinical examination, and sonographic mapping. Myomas were enucleated and retrieved laparoscopically. Myoma beds were sutured in multiple layers by endoscopic intracorporeal suturing. Fluid as adhesion barrier was used.

Results: Three hundred and eighty two patients presented with subfertility, 69 with menorrhagia, and 59 with abdominal mass. The average maximum diameter of myoma was $9.1 \mathrm{~cm}$. The mean duration of surgery was 90 minutes. The mean postoperative stay was 24 hours. No intraoperative complication occurred and the hospital course was uncomplicated. In seven cases, minilap incision was given for retrieval of myoma and suturing of the bed. Two patients had minor delayed wound healing of the morcellator port site. The patients did not report any complaints during follow-up except one patient who developed omental hernia at morcellator port site. There was no rupture of scar and very low adhesion scores in subsequent cesarean sections or second look scopies.

Conclusion: With proper multilayer closure of the myoma bed, laparoscopic myomectomy is feasible for moderate and even large myomas and has good outcomes in terms of fertility and alleviation of symptoms.

Keywords: Better reproductive outcome, Laparoscopic myomectomy, Large myomas, Multilayer closure.

How to cite this article: Jain N, Singh S. Multiple-layer Closure of Myoma Bed in Laparoscopic Myomectomy. Int J Gynecol Endsc 2017;1(1):22-27.

Source of support: Nil

Conflict of interest: None

${ }^{1}$ Director, ${ }^{2}$ Clinical Research Fellow

1-2Department of Gynecology and Obstetrics, Vardhman Trauma \& Laparoscopy Center Pvt. Ltd., Muzaffarnagar, Uttar Pradesh, India

Corresponding Author: Nutan Jain, A-36, South Civil Lines Mahavir Chowk, Muzaffarnagar, Uttar Pradesh, India, Phone: +911312623084, e-mail: Jainnutan@gmail.com

\section{INTRODUCTION}

Uterine leiomyoma is the most common tumor of female reproductive tract. An incidence of 20 to $25 \%$ in sexually active and 30 to $35 \%$ in all women irrespective of age has been reported. ${ }^{1}$ The surgical management of myomas has advanced significantly, and there has been a renewed interest in the removal of myoma alone, i.e., myomectomy rather than hysterectomy, which was carried out historically. With the trends toward minimally invasive endoscopic surgery, procedures have been developed to accomplish myomectomy via either laparoscopic or hysteroscopic route. ${ }^{2}$ Just as these procedures have come up, they have met with several criticisms like lack of meticulous closure, inadequate hemostasis, chances of rupture in subsequent pregnancy, and high risk of adhesion formation. We report our experience of 510 cases of laparoscopic myomectomy of large and moderate size myomas in a tertiary care hospital.

\section{AIMS AND OBJECTIVES}

In this study, our objective is to describe the technique of laparoscopic myomectomy and multiple-layer closure of myoma bed and discuss its outcomes and advantages, overcoming all the criticisms surrounding laparoscopic myomectomy.

\section{MATERIALS AND METHODS}

In a retrospective study, conducted at our tertiary care hospital, we evaluated 510 patients in the past 6 years with large and moderate size myomas, largest being up to $22 \mathrm{~cm}$ producing an abdominal mass corresponding to 36 weeks size uterus.

Inclusion criteria included largely:

- Patients being treated for subfertility with myomas causing endometrial distortion or tubal occlusion with normal ovulation and normal male factor.

- Patients with large myomas producing pressure symptoms.

- Asymptomatic large myomas producing palpable abdominal mass up to the umbilicus or above.

Preoperative evaluation included history, clinical examination, basic investigation for preanesthetic checkup, and a detailed transvaginal scan. Sonography included mapping of myomas in number, size, and 
location, and most importantly, differentiating it from adenomyosis and adenomyoma. ${ }^{3}$ Although we carry out adenomyomectomy with almost identical technique, still surgical preparedness and patient counseling are done accordingly. In fast growing and large myoma, we also carry out serum lactate dehydrogenase, magnetic resonance imaging, and color flow indices to rule out sarcoma. ${ }^{4}$

In all cases, an informed consent was taken. Hemoglobin was optimized using iron sucrose injections. Extensive bowel preparation was carried out in each case. No patient received gonadotropin-releasing hormone analog prior to surgery as it leads to loss of cleavage plane during surgery. Ulipristal and mifepristone were also not used in preoperative period to decrease the size of myoma. Lately, we found that pretreatment with these two medical agents was also associated with cystic degeneration and difficulty in enucleating myoma. ${ }^{5,6} \mathrm{All}$ operations were performed under general anesthesia with inhalational anesthetics avoiding nitrous oxide as it causes bloating of bowel. ${ }^{7}$

In larger myomas, about two hours before the start of surgery, misoprostol $400 \mathrm{~g}$ was given per rectal to prevent blood loss. Oxytocin 20 units in $1 \mathrm{~L}$ of normal saline solution is started at $40 \mathrm{mU} / \mathrm{min}$. Injection tranexamic acid two ampoules IV was also given. All these agents were given in an endeavor to reduce blood loss ${ }^{8-10}$ in cases of large myomas.

Our innovative technique of port placement involved intraumbilical Veress needle insertion without any incision and utilizing right upper $5 \mathrm{~mm}$ paraumbilical incision for primary trocar insertion. The $10 \mathrm{~mm}$ port was optimized supraumbilically, under direct vision of $5 \mathrm{~mm}$ telescope depending on the myoma size, which went even up to the xiphisternum in large myomas. All accessory ports are also made so that they remain above and outside the biggest myoma. The most common port placement is two ipsilateral ports for enucleation and suturing, one contralateral port for the assistant anywhere between umbilicus and anterior superior iliac spine (depending on myoma size) in a line drawn $2.5 \mathrm{~cm}$ drawn medial to anterior superior iliac spine (Fig. 1). Additionally, suprapubic and right paraumbilical port can be employed for bigger and multiple myoma. In cases of previous laparotomy or laparoscopy, we employ the "Jain point"11 to gain access for the primary $5 \mathrm{~mm}$ port and telescope. Jain point is right paraumbilical port on a line drawn $2.5 \mathrm{~cm}$ medial to anterior superior iliac spine. Rather than using the palmar point, we use Jain point as it is more lateral, so it moves further away from previous incision. It is more ergonomic to use and become the main working port in the due course of surgery. We have used it in very severe cases of big and bad surgical scars of intestinal obstruc-

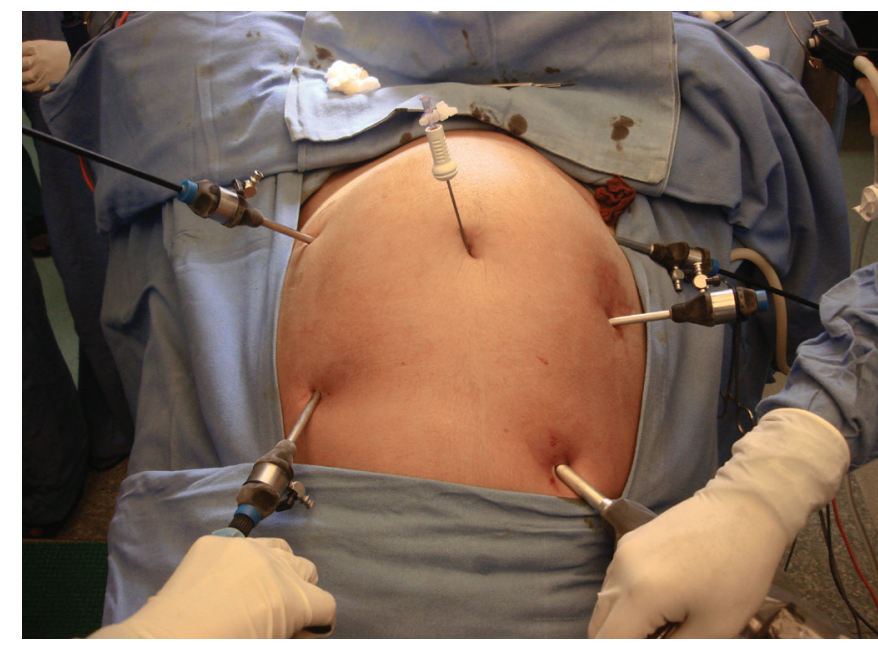

Fig. 1: Port placement (right- and left-sided ipsilateral ports. Veress needle left in place sprinkling the operative field with saline as needed)

tion, previous cesarean section and myomectomies. In this series, we have not resorted to palmar point in either of the cases, ${ }^{12}$ though at least one-third of the cases had a previous laparotomy or laparoscopy.

On peritoneal entry, all pelvic and abdominal structures were inspected and other pathologies, if present, were noted. Vasopressin was injected at a concentration of 20 units $/ 200 \mathrm{~mL}$ between serosa and pseudocapsule of myoma to decrease the bleeding and for hydrodissection. For big myomas we injected up to 400 to $600 \mathrm{~mL}$ to facilitate hydrodissection in an avascular plane. Hysteroscopy was carried out in the meantime to see and treat any submucous myoma.

In most of the cases, we preferred to make a transverse incision over the most bulging part of the myoma with the harmonic scalpel, taking care not to extend to cornual ends. In case of multiple myomas, we preferred to give single anterior or posterior incision in such a manner that most myomas can be enucleated by a single incision from superficial to deepest locations. If incision was expected to extend too far, then vertical incision was resorted to very rarely. Sharp dissection was carried out in the plane between myoma and the pseudocapsule with harmonic scalpel, coagulating and cutting all vascular bridges, causing very minimal blood loss. Two myoma screws were used in case of very large myomas, one was kept in the constant position from the suprapubic port and the other kept changing to allow for traction near the working end. Myoma was finally enucleated from its bed by traction and countertraction. After enucleation, there was usually no bleeding, but if at all there was, then light minimal bipolar coagulation was carried out. After achieving adequate hemostasis, myoma bed was sutured in transverse, continuous, and nonlocking fashion in multiple layers. A suture of 45 to $50 \mathrm{~cm}$ length, no. 1-0 
vicryl on a taper cut needle was loaded and introduced in the peritoneal cavity using the Clark Reich method of removing the $5 \mathrm{~mm}$ trocar. The angle knot was taken by passing the suture from upper and lower edge of myoma bed and an intracorporeal, tight surgeon's knot was secured. From this point continuous, nonlocking suturing was carried out using needle holders in both hands for a much faster and better grip in suturing. First layer of deep myometrium was thus completed, and the same suture is used to take second layer of superficial myometrium. Third layer of serosa was taken, up to the starting point of the first layer at the angle and tied there in very large myomas, which were very deep intramural and cause a symmetrical enlargement of the uterus; the myoma bed, which comes after myomectomy was very deep. So, we preferred to go from the deepest myometrium working upward layer by layer till a total reconstruction is carried out, and uterus appears absolutely in shape with only one knot on the surface. In very large myomas, we sutured in even up to six layers for proper approximation of the defect. While suturing all the layers, care was taken to take bites at equal distances and take adequate tissue to fully obliterate all dead space and give a neat look. To minimize the exposed thread volume, we also apply subserosal baseball sutures in most superficial layers. In this way, there was only one knot in the entire myoma bed, minimizing the knot volume and decreasing the adhesion formation potential. Barb sutures were used with equal frequency and have been found to give good approximation, tensile strength, and obliteration of myoma bed for bigger and deep myomas. ${ }^{13}$ To avoid the occasional untoward side effect of subacute intestinal obstruction due to adhesion of bowel on the exposed barb suture line, ${ }^{14}$ we prefer to do the deeper layers with barb suture and to cover up the most superficial layer with 2-0 vicryl (Figs 2A to K). This has worked very well as in this series we did not get even a single case of bowel obstruction, and adhesion score remained the same. Copious irrigation and lavage were carried out. In young patients and unsuspicious myomas, retrieval of tissue is done by Rotocut G1 morcellator (Karl storz, Germany) via $15 \mathrm{~mm}$ sleeve. Morcellator port site is meticulously closed by port closure needle. Contained bag morcellation, ${ }^{15}$ posterior colpotomy, and laparoscopic-assisted myomectomy (LAM) are also used in selected cases. Instillation of Icodept $4 \%$ (Icodextrin) or lactate solution $2 \mathrm{~L}$ was done at the end of procedure for hydroflotation and to prevent adhesion formation. We have not used surface adhesion barriers and used fluid barriers.

All patients were up and about within 4 hours of surgery, were orally allowed, started ambulation and deep breathing and leg raising exercise, and were discharged after 24 hours.

\section{RESULTS}

The mean age of patients was $30 \pm 2$ years. Three hundred and eighty two $(74.9 \%)$ patients presented with subfertility, 69 (13.55\%) patients with menorrhagia, and 59 (11.55\%) patients with abdominal mass. The average maximum diameter of the myoma was $9.1 \mathrm{~cm}$.

The mean duration of surgery was 90 minutes. No intraoperative complication occurred. In seven cases, minilap incision was given for retrieval of myomas and suturing of myoma bed. There was, in fact, a case of large uterus with multiple myomas, which made a pure laparoscopic approach difficult due to lack of plane of cleavage, leading to more bleeding and difficult closure in adenomyotic tissue. Blood transfusion was required only in $51(10 \%)$ patients with very large size myomas or multiple myomectomy sites or with previously low hemoglobin levels. There were no conversions to laparotomy ever and never a hysterectomy. In one of the case with previous myomectomy and 34-week-size uterus, she had dense bowel and omental adhesion. After 24 hours, patient presented with hemoperitoneum necessitating insertion of scope and picking the oozer found in omentum, which was separated from previous adhesion.

The mean postoperative stay was 24 hours. All patients had an uneventful recovery. None of the patients had reactionary or late secondary hemorrhage.

Three patients reported delayed wound healing of the morcellator site and one patient developed omental hernia at morcellator site on long-term follow-up.

Very low adhesion scores were observed and in subsequent second look, scopies carried out by us. Other colleagues also reported very low adhesions in patients they delivered by cesarean section.

Among the subgroup of patients who presented with infertility, 268 (70\%) patients conceived after the surgery had an uneventful antenatal and intranatal course. No patient had rupture of myomectomy scar. Minimal adhesions were noted in subsequent cesarean sections. Even the first myomectomy we did conceived after 17 years of married life, just 1 month after myomectomy. She continued pregnancy up to term and an elective cesarean was carried out at term; no adhesions or even depression at myomectomy site was observed.

Recurrence of myomas was seen in $51(10 \%)$ patients. Eight $(1.6 \%)$ patients out of these, who were around 35 years of age when myomectomy was performed for menorrhagia, later underwent hysterectomy. Three $(0.6 \%)$ patients desirous of pregnancy had a repeat myomectomy carried out on account of recurrence of myoma during the study period. 

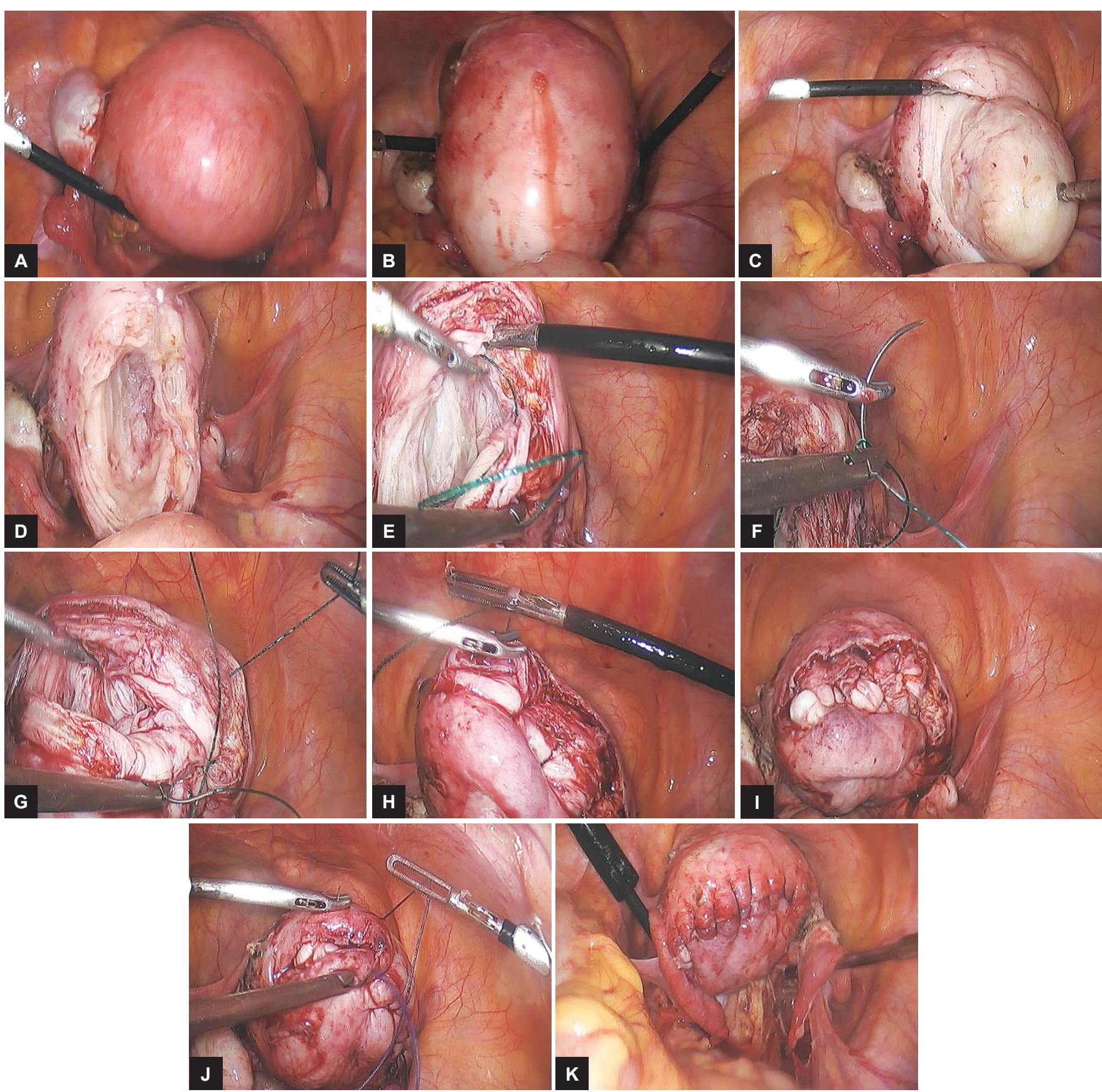

Figs 2A to K: (A) Big posterior wall myoma; (B) blanched look of myoma after injecting diluted vasopressin; (C) enucleation of myoma; (D) myoma bed after myoma enucleation; $(E)$ needle passing through posterior edge; $(F)$ needle passing through loop of V-Loc suture; (G) first layer in progress with V-Loc suture; $(\mathrm{H})$ second layer in progress; $(\mathrm{I})$ third layer completed; $(\mathrm{J})$ last layer incorporating subserosa and serosa with vicryl; and $(\mathrm{K})$ final appearance of myoma bed sutured

\section{DISCUSSION}

In this era of minimally invasive endoscopic surgery, laparoscopic myomectomy has become the order of the day. It offers several advantages over laparotomy, including less operative trauma and blood loss, reduced postoperative morbidity, shorter hospital stay and recovery time, earlier return to normal activity, fewer postoperative adhesions, better cosmesis, improved patient compliance, and better pregnancy outcome. Furthermore, the magnification it provides allows careful microsurgical dissection, development of avascular planes, and perfect hemostasis. ${ }^{16}$
Many surgeons still prefer to do open myomectomy due to technical difficulties or lack of endosuturing skills. One of their major concerns is the risk of hemorrhage and uterine rupture in the subsequent pregnancies. ${ }^{17}$ It has been reported that rupture can occur during the course of pregnancy or during delivery after removal of myomas.

However, in the experienced surgeon's hands, with superior skill of endosuturing, laparoscopic myomectomy is a very safe procedure and such complications are rare.

A long-term survey by Dubuisson et $\mathrm{al}^{18}$ found three cases of spontaneous uterine rupture in 15 pregnancies 
and only one occurred at the laparoscopic myomectomy site. The strength of scar largely depends upon proper meticulous closure of the incision site. That is why multiple-layer closure gives much better results than single- or two-layer closures. It also gives much better approximation of the defect and a neat finish at the end of procedure. Based on the clinical trials and case series, it would appear that the risk of uterine rupture during pregnancy is no higher than $1 \%$ when the myomectomy incision is appropriately repaired. ${ }^{19}$ We had no scar rupture even in very big myomas or multiple myomas.

Another complication causing much concern is the subsequent adhesion formation. We, at our institute, perform continuous suturing of the myoma bed using no. 1-0 polygalactin suture on taper cut needle in multiple layers so that there is only one knot on the surface. The amount of adhesions formed per suture line is directly proportional to the number of knots, and lesser the number of bulky knots lesser the adhesion scores. We have observed that this technique gives much better results than that of interrupted knotting, leading to tremendous knot volume. However, this requires advanced suturing skills.

Retrieval of large myoma also poses technical difficulties to most of the surgeons. Morcellators have made this task much simpler. ${ }^{20}$ As the myoma is progressively morcellated and the uterine size reduced, additional space is created for the optimum movement of instruments. The 12 or $15 \mathrm{~mm}$ claw forceps of the morcellator offers better grip and steady traction. This tremendously reduces the operating time and reduces the technical difficulty of the procedure. We took extreme care to remove myomas in longer chips to reduce the morcellation time. Exact count of myomas and location of their parking in the abdomen was kept to avoid misplacing any myoma. Such misplaced myomas are a common cause of iatrogenic myomas as reported by Nezhat and $\mathrm{Kho}^{21}$ in their study.

The only complication we observed was that of delayed wound healing of the morcellator site in two patients. This was probably due to jagged margins created by repeated manipulations at the morcellator site. We thus recommend that one should avoid using towel clips to avoid gas escape around morcellator site, which starts off in prolonged morcellation time.

Another safer and relatively simpler procedure in the hands of experienced laparoscopists as well as novices is laparoscopic-assisted minilap myomectomy. ${ }^{22}$ In this procedure, initially laparoscopy is performed giving incision over myoma with the ultrasonic shears. Myoma is partly enucleated by traction of myoma screw. A 3 to $4 \mathrm{~cm}$ minilap Pfannenstiel incision is given. Through this minilap incision, enucleation and suturing of beds is carried out of all multiple and big myomas. Then minilap incision is sutured back, laparoscope reinserted, and thorough suction irrigation and lavage was carried out. However, it should be planned preoperatively and there is no sudden conversion on table from laparoscopic myomectomy to LAM.

In recent times, some controversies regarding morcellation prompted us to revisit other routes of myoma retrieval. Posterior colpotomy and LAM both aim at reintroducing alternate methods of removing myomas other than morcellation. Contained bag morcellation is also done in select cases of fast growing myomas, though the Association of Gynecologic Laparoscopists ${ }^{23}$ has concluded that there exist insufficient data to discontinue power morcellation in low-risk, appropriately screened patients.

Our experience demonstrates the feasibility of dealing with even large size myoma successfully laparoscopically, and we encourage multiple-layer closure to decrease the dead space and avoidance of hematoma formation and, in turn, achieve good reproductive outcome.

\section{CONCLUSION}

Laparoscopic myomectomy is a safe and favorable alternative to open myomectomy as it offers several advantages over laparotomy with minimal complications. ${ }^{24}$ It is equally feasible for even large size myomas in experienced hands with advance suturing skills. Multilayer closure of the myoma bed in continuous, nonlocking fashion gives excellent reproductive outcomes in terms of very low adhesion scores and negligible risk of scar rupture in subsequent pregnancies. However, one should appropriately select the myoma size according to one's suturing skills, instruments available, and experience.

\section{REFERENCES}

1. Buttram VC Jr, Reiter RC. Uterine leiomyomata: etiology, symptomatology and management. Fertil Steril 1981 Oct;36(4):433-445.

2. Hurst BS, Mathews ML, Marshburn PB. Laparoscopic myomectomy for symptomatic uterine myomas. Fertil Steril 2005 Jan;83(1):1-23.

3. Fedele L, Bianchi S, Dorta M, Arcaini L, Zanotti F, Carinelli S. Transvaginal sonography in the diagnosis of diffuse adenomyosis. Fertil Steril 1992 Jul;58(1):94-97.

4. Goto A, Takeuchi S, Sugimura K, Maruo T. Usefulness of Gd-DTPA contrast-enhanced dynamic MRI and serum determination of $\mathrm{LDH}$ and its isozymes in the differential diagnosis of leiomyosarcoma from degenerated leiomyoma of the uterus. Int J Gynecol Cancer 2002 Jul-Aug;12(4):354-361.

5. DonnezJ, TatarchukTF, Bouchard P,Puscasiu L, Zakharenko NF, Ivanova T, Ugocsai G, Mara M, Jilla MP, Bestel E, et al.; PEARL I Study Group. Ulipristal acetate versus placebo for fibroid treatment before surgery. N Engl J Med 2012 Feb;366: 409-420. 
6. Engman M, Granberg S, Williams AR, Meng CX, Lalitkumar PG, Gemzell-Danielsson K. Mifepristone for treatment of uterine leiomyoma. A prospective randomized placebo controlled trial. Hum Reprod 2009 Aug;24(8):1870-1879.

7. Alexander GD, Noe FE, Brown EM. Anesthesia for pelvic laparoscopy. Anesth Analg 1969 Jan-Feb;48(1):14-18.

8. Fletcher H, Frederick J, Hardie M, Simeon D. A randomized comparison of vasopressin and tourniquet as hemostatic agents during myomectomy. Obstet Gynecol 1996 Jun;87(6):1014-1018.

9. Celik H, Sapmaz E. Use of a single preoperative dose of misoprostol is efficacious for patients who undergo abdominal myomectomy. Fertil Steril 2003 May;79(5):1207-1210.

10. Caglar GS, Tasci Y, Kayikcioglu F, Haberal A. Intravenous tranexamic acid use in myomectomy: a prospective randomized double-blind placebo controlled study. Eur J Obstet Gynecol Reprod Biol 2008 Apr;137(2):227-231.

11. Jain N, Sareen S, Kanawa S, Jain V, Gupta S, Mann S. Jain point: a new safe portal for laparoscopic entry in previous surgery cases. J Hum Reprod Sci 2016 Jan-Mar;9(1):9-17.

12. Palmer R. Safety in laparoscopy. J Reprod Med 1974 Jul;13(1): 1-5.

13. Einarsson JI, Chavan NR, Suzuki Y, Jonsdottir G, Vellinga TT, Greenberg JA. Use of bidirectional barbed suture in laparoscopic myomectomy: evaluation of perioperative outcomes, safety, and efficacy. J Minim Invasive Gynecol 2011 Jan-Feb; 18(1):92-95.

14. Kindinger LM, Setchell TE, Miskry TS. Bowel obstruction due to entanglement with unidirectional barbed suture following laparoscopic myomectomy. Gynecol Surg 2012 Oct;9(3):357-358.

15. Venturella R, Rocca M, Lico D, La Ferrera N, Cirillo R, Gizzo S, Morelli M, Zupi E, Zullo F. In-bag manual versus uncontained power morcellation for laparoscopic myomectomy: randomized controlled trial. Fertil Steril 2016 May;105(5):1369-1376.
16. Reich H, Thompson KA, Nataupsky LG, Grabo TN, Sekel L. Laparoscopic myomectomy: an alternative to laparotomy myomectomy or hysterectomy? Gynecol Endosc 1997 Feb;6(1):7-12.

17. Dubuisson JB, Chapron C, Levy L. Difficulties and complications of laparoscopic myomectomy. J Gynecol Surg 2009 Feb;12(3):159-165.

18. Dubuisson JB, Fauconnier A, Deffarges JV, Norgaard C, Kreiker G, Chapron C. Pregnancy outcome and delivery following laparoscopic myomectomy. Hum Reprod 2000 Apr;15(4):869-873.

19. Sinha R, Sundaram M. Laparoscopic management of large myomas. J Gynecol Endosc Surg 2009 Jul;1(2):73-82.

20. Sinha RY, Hegde A, Warty N, Jain R. Laparoscopic devascularization of uterine myomata followed by enucleation of the myomas by direct morcellation. J Am Assoc Gynecol Laparosc 2004 Feb;11(1):99-102.

21. Nezhat $C$, Kho K. Iatrogenic myomas: a new class of myomas. J Minim Invasive Gynecol 2010 Sep-Oct;17(5):544-550.

22. Nezhat C, Nezhat F, Bess O, Nezhat CH, Mashiach R. Laparoscopically assisted Myomectomy: a report of a new technique in 57 cases. Int J Fertil 1994 Jan-Feb;39(1):39-44.

23. AAGL statement on power morcellation - The following statement was authored by Dr. Jubilee Brown, a member of the AAGL Board of Trustees and summarizes the presentation she made on behalf of the AAGL to the Obstetrics and Gynecology Devices Panel of the FDA Medical Devices Advisory Committee on July 11, 2014. The statement was approved by the Board of Trustees http:/ / www.aagl.org/aaglnews/aaglstatement-to-the-fda-on-power-morcellation/

24. Miller CE, Davies S, Johnston M. Laparoscopic myomectomy with uterine reconstruction is a safe surgical procedure. Presented at the 27th Annual Meeting of the American Association of Gynecologic Laparoscopists. Atlanta, GA. 1998. 